\title{
Educazione interculturale
}

De la différence à la coexistence

\section{Gloria Paganini}

\section{CpenEdition}

\section{Journals}

Édition électronique

URL : http://journals.openedition.org/ries/2962

DOI : $10.4000 /$ ries.2962

ISSN : 2261-4265

Éditeur

Centre international d'études pédagogiques

Édition imprimée

Date de publication : 1 mars 1998

Pagination : 119-132

ISBN : 9771254459005

ISSN : 1254-4590

Référence électronique

Gloria Paganini, "Educazione interculturale », Revue internationale d'éducation de Sèvres [En ligne],

17 | 1998, mis en ligne le 19 avril 2013, consulté le 19 avril 2019. URL : http://journals.openedition.org/ ries/2962 ; DOI : 10.4000/ries.2962

Ce document a été généré automatiquement le 19 avril 2019

(c) Tous droits réservés 


\title{
Educazione interculturale
}

\author{
De la différence à la coexistence
}

\author{
Gloria Paganini
}

1 L'attitude positive et ouverte envers l'étranger, l'aptitude à établir avec lui des rapports d'échange et d'intercompréhension s'enracinent dans l'éducation familiale, mais c'est à l'école que revient, avant toute chose, de relayer la famille dans l'éducation à la diversité : «Elle a à exercer un pareil rôle, désormais » comme le rappelle Louis Porcher, «à l'égard des nouvelles instances d'inculcation, les médias notamment $»^{1}$.

2 Mais que signifie «enseigner la diversité à l'école ?» Est-ce élaborer un projet éducatif dans lequel la diversité serait appréhendée sous forme de contenus spécifiques, au sein d'une discipline donnée, ou apprendre à intégrer l'évidence - ou celle qui devrait en être une - que chacun incarne l'altérité de quelqu'un d'autre, et réciproquement? On ne peut aujourd'hui qu'« interroger le concept même d'altérité dans sa double relativité, écrit Marc Augé, puisque les autres aussi définissent l'autre $»^{2}$.

3 L'Italie, comme chacun sait, s'est rapidement transformée de pays d'émigration en pays d'immigration: dans les dix dernières années la multiplication d'ouvrages qui s'interrogent sur l'incidence de la nouvelle réalité multiculturelle sur le système éducatif signale l'ampleur des choix auxquels société et école se mesurent, non sans difficultés. La récurrence du qualificatif inter culturale dans la production éditoriale italienne atteste l'émergence et la vitalité d'un questionnement que l'on pourrait résumer de la manière suivante : comment penser la différence pour qu'elle puisse rendre possible la coexistence ${ }^{3}$ ?

4 Avant d'être progressivement intégrées, les populations immigrées ont toujours été perçues dans un premier temps comme susceptibles de porter atteinte à l'existence et à l'intégrité de la communauté d'accueil. On rappellera, à titre d'exemple, qu'en Suisse au tout début du siècle «les étrangers qui conservent leurs mœurs, leurs usages, leurs aspirations propres sont censés opérer un travail de "dénationalisation" des valeurs autochtones. Certaines nationalités, les Italiens en particulier, font l'objet d'une stigmatisation raciste qui attaque l'individu dans ses habitudes quotidiennes, alimentaires, vestimentaires, hygiéniques, récréatives, sociales ${ }^{4} »$. 


\section{Italie : la distance intérieure}

Ce concept d'altérité, comment est-il interrogé dans un pays comme l'Italie, qui se découvre multiethnique, après avoir incarné aux yeux des voisins les plus proches, la figure même de la différence irréductible ? Son cas semble bien constituer un exemple de la « double relativité » qui traverse la réflexion contemporaine, puisque ceux-là mêmes qui dans un passé récent étaient perçus comme « les autres » redéfinissent à présent leur propre identité en fonction de ces nouveaux «autres " qu'ils perçoivent à leur tour comme « très différents ».

6 Nous nous proposons d'aborder cette problématique en prenant appui sur trois ouvrages parus en Italie respectivement en 1992, 1993 et $1996^{5}$ qui, mettant au centre du débat la notion d'educazione interculturale, partagent la même préoccupation d'accompagner d'une réflexion critique le renouvellement en cours dans la culture éducative et institutionnelle italiennes.

7 Les diverses contributions à ces ouvrages se rejoignent dans une aspiration commune : éduquer à l'altérité signifie dépasser le zèle purement humanitaire, les vocations iréniques et l'attitude naïvement philanthropique. Ainsi, d'une part, la nécessité s'impose de passer de l'educazione interculturale à la pedagogia interculturale ${ }^{6}$, c'est-à-dire de rendre plus incisive l'action éducative en l'inscrivant dans un cadre épistémologique capable d'en consolider et d'en orienter le projet. D'autre part, l'urgence est ressentie d'adapter le cadre législatif à la nouvelle fonction qui est assignée à l'école, celle de médiatrice entre des cultures différentes.

8 Les premières interventions officielles en matière d'éducation interculturelle datent, en Italie, de 1989 et $1990^{7}$; les objectifs sont précisés en 1992 dans une circulaire émanant du ministère de l'Instruction publique ${ }^{8}$, dont nous résumons ici les principales orientations.

9 Trois grandes transformations - mondialisation, processus d'unification européenne, nouveaux phénomènes migratoires - soulignent l'urgence d'élaborer de nouvelles formes de contact et d'échange. L'école, qui constitue à la fois le cadre et le facteur stratégique de cette élaboration, est conviée à promouvoir un nouvel équilibre entre identité nationale et cultures "autres», équilibre qui doit être entendu non pas comme simple juxtaposition entre cultures, mais comme «coprésence, réciprocité, dialogue et échange ». À l'école, «il est demandé en particulier de doter les nouvelles générations d'outils aptes à combattre, sur le plan intellectuel, culturel, éthique, religieux et psychologique, les stéréotypes qui exaspèrent les conflits et éloignent les espoirs de paix ${ }^{9}$ ».

Les objectifs de l'éducation interculturelle seront donc les suivants :

- amener à la compréhension des processus à travers lesquels se construit une culture, qu'il s'agisse de la culture native ou des cultures étrangères avec lesquelles on entre en contact ;

- favoriser l'élaboration et l'appropriation - individuelle et collective - des valeurs sur lesquelles repose le droit de chacun au respect de sa propre histoire et à la construction d'une histoire commune ;

- faciliter l'acquisition de savoirs et d'outils qui aident à interagir avec les Autres, en assumant une attitude solidaire envers chaque personne et, notamment, envers les moins favorisées. 
11 La réglementation actuelle s'inscrit ainsi dans la continuité du cadre législatif qui incitait, dès les années soixante-dix, au respect du « pluralisme culturel »: mais il s'agissait pour l'époque d'une priorité visant à faire reconnaître et respecter les différences internes au pays, différences régionales, sociales et linguistiques ; si cette priorité garde aujourd'hui sa validité de principe, elle s'est néanmoins complexifiée sous les effets de l'internationalisation et sous l'impulsion des nouveaux phénomènes migratoires. La présence accrue sur le territoire italien de groupes appartenant à des cultures «très différentes de celles auxquelles le pluralisme de notre pays nous avait habitués ${ }^{10}$ " est toutefois à considérer comme une situation privilégiée, condition d'un enrichissement mutuel, dans la compréhension et le respect des identités réciproques.

La distance culturelle ne serait ainsi que la dernière en date des différences auxquelles l'école italienne se mesure. La dernière, et peut-être la plus difficile à appréhender, mais non la seule, puisque, comme l'évoque Alessandra Durino Allegra, des situations conflictuelles persistent entre Italiens provenant de régions différentes. De surcroît, il ne faut pas oublier les problèmes liés à la diversité culturelle interne qui n'ont jamais été définitivement réglés par l'unification géographique et politique de l'Italie ${ }^{11}$.

D'ailleurs, au moment même où les premiers textes officiels concernant l'éducation interculturelle assignaient à l'école la tâche de valoriser les cultures «autres » - y compris, précise encore la circulaire, celles qui dans le passé auraient été perçues « comme exotiques ou irréductiblement ennemies » - une nouvelle réglementation était discutée au Parlement italien, visant à protéger les minorités linguistiques, particulièrement nombreuses, sur le territoire national ${ }^{12}$. A l'égard de la diversité, de quelque nature qu'elle soit, écrivait alors Raffaele Simone, on ressent un sentiment d'embarras. Faute de pouvoir assumer une position unitaire, « on en vient à accepter des positions pluralistes au-delà de toute logique et faisabilité13 ». Alors que la population italienne avance à travers maints détours vers l'acquisition d'une langue qui soit la même pour tous, peut-on assigner une charge supplémentaire à une école qui, déjà, assure si laborieusement sa tâche principale de doter tout le monde d'un patrimoine commun suffisant?

\section{Spécificité italienne et éducation à la diversité}

L'arrière-plan de ce débat signale la complexité de la situation italienne : dans l'Union européenne, l'Italie est le pays au plus grand nombre de minorités linguistiques, presque le double de celles que compte la France, classée deuxième ${ }^{14}$. En outre - peut-être est-il utile ici de le rappeler - l'italophonie concernait seulement au moment de l'Unité nationale (1861) 2,5\% des Italiens et arrivait à $30 \%$ de la population cent ans plus tard. En 1974, plus de la moitié des Italiens parlaient un dialecte à la maison et 23,9\% d'entre eux alternaient l'italien et le dialecte en $1982^{15}$. Il est bien connu par ailleurs que la diffusion des médias, davantage que l'école, a imprimé l'impulsion la plus significative, sinon définitive, au processus d'unification linguistique et culturelle de l'Italie.

Dans un tel contexte de pluralité intra-nationale, confrontée aux différences culturelles introduites par la densité et l'hétérogénéité des mouvements migratoires, la question est alors de savoir comment l'on pourrait ne pas éduquer à la diversité à l'école en Italie. Le cas de deux élèves de l'école primaire italienne, que nous empruntons à la revue Italiano \& Oltre ${ }^{16}$, nous paraît exemplaire de la spécificité italienne. 
17 Jorgu et Violeta sont deux jeunes Albanais, respectivement de quatorze et de onze ans, arrivés en Italie lors de l'exode de 1991 et, depuis, régulièrement inscrits à l'école italienne dans une région du sud de l'Italie. Deux ans après leur arrivée, Jorgu et Violeta se situent aux deux extrêmes de l'éventail des comportements adoptés par les jeunes Albanais. Jorgu montre une préférence marquée et presque absolue pour le dialecte local. Lorsqu'il a recours à l'italien, il produit des phrases très brèves et simplifiées. Violeta parle peu, mais choisit toujours l'italien, sauf les rares occasions où elle alterne dialecte et italien avec ses camarades. Les deux langues disponibles pour les élèves albanais, comme pour n'importe quel élève natif de cette ville, sont donc le dialecte et l'italien. Ces élèves se sont rapidement appropriés les règles d'usage des deux langues en contact et, comme tout natif, ils alternent intuitivement deux langues de statut différent (l'italien étant la langue de prestige, le dialecte, celle de la complicité avec les pairs), en fonction de la situation et des interlocuteurs. Maria Teresa Romanello souligne à juste titre l'incidence et la valeur de cette compétence dans le processus d'intégration de ces élèves dans la société d'accueil. Les variations dans l'application de cette compétence sociolinguistique ne peuvent s'expliquer, dans le cas de Jorgu notamment, qu'en rappelant son histoire. Né dans une famille très pauvre de Durazzo, à douze ans il prend le bateau pour l'Italie où, après une série de vicissitudes, il est confié à un couple italien. Lorsqu'on l'incite à parler italien, il déclare avoir le devoir d'utiliser la langue de son père, le dialecte. Cette loyauté linguistique, comme l'appelle l'auteur, que justifie donc un très profond sentiment de gratitude à l'égard du père adoptif, constitue chez Jorgu une autre forme de compétence, non entièrement réductible, nous semble-t-il, à une simple conscience d'ordre métalinguistique.

L'éducation à la diversité, comme on peut le constater, ne saurait se concevoir comme un face-à-face entre une réalité uniforme, homogène d'un côté et, de l'autre, la différence, l'altérité, la diversité. C'est une réalité italienne plurielle - depuis longtemps traversée par une double vocation à sauvegarder les différences et à consolider le processus d'unification - qui se mesure aujourd'hui à la nécessité (et à l'opportunité) d'appréhender les effets des phénomènes migratoires, qui ont brusquement pris des dimensions auxquelles les individus, tout comme les institutions, n'étaient guère préparés.

En outre, la singularité de la situation nationale s'inscrit dans une mutation de nature, cette fois, planétaire, qui renforce le degré d'interdépendance entre les constituants d'un même système mondial. La nôtre, écrit Fulvio Poletti, est une "société complexe et fluide (...) qui a perdu le "centre", dissous dans une organisation en forme de rhizome, où chaque point de la trame du tissu socioculturel peut être simultanément "centre" et "périphérie"17 ». L'intensification exponentielle de la communication et de la mobilité internationales, la mise en relation avec des communautés et des cultures aussi lointaines que diversifiées, exige « un modèle flexible et mobile d'identité et d'autoréalisation ${ }^{18}$ ».

Il est clair qu'une tâche si ardue et si complexe ne peut être assignée à l'école qu'à la condition que soient en même temps mobilisées vers le même objectif toutes les autres instances et ressources éducatives et formatrices. L'école a toutefois un rôle central et spécifique à jouer, celui de doter les jeunes sujets «de systèmes de pensée et de procédures de catégorisation flexibles et ouvertes et non rigides et sclérosés, autour de schémas univoques et immuables $»^{19}$ où l'autre s'opposerait au soi, où l'étranger et l'inconnu seraient automatiquement assimilés à un danger pour le nous. Assumant ce rôle, l'école s'institue en lieu privilégié pour la promotion d'une "éducation à la mondialité $»^{20}$, poursuivant des finalités qui sont, sinon exhausives, néanmoins capitales : dépassement 
du processus de stéréotypisation, relativisation de sa propre position par la mise en relation et par la comparaison avec celle des autres, acquisition d'une aptitude relationnelle et solidariste, notamment par les échanges et les coopérations interrégionales et internationales. Ainsi, une véritable "éducation à la mondialité » correspond à une "éducation à la différence», qui, au sein d'un processus vraisemblablement inéluctable d'uniformisation du village global, puisse amener à découvrir et à valoriser précisément les identités, les valeurs, les spécificités qui rendent chacun original, c'est-à-dire différent ${ }^{21}$.

L'entreprise est d'autant moins simple que les résistances majeures sont de nature irrationnelle et inconsciente : d'où la nécessité, souligne encore Fulvio Poletti, d'élaborer un projet éducatif non exclusivement intellectuel, mais capable d'incorporer les composantes intuitives et affectives, une éducation, donc, centrée à la fois sur la connaissance et la compréhension, visant en même temps la plasticité cognitive et la qualité émotionnelle de l'écoute et de l'empathie ${ }^{22}$.

Une tâche si complexe, si urgente, ne saurait non plus être confiée à une seule discipline : l'educazione interculturale devra avant tout être intégrée de manière transversale au curriculum scolaire existant ${ }^{23}$. Une transversalité qui peut être entendue soit comme choix d'un fil conducteur (thématique ou méthodologique) simultanément abordé par les différentes disciplines, soit comme approche critique des disciplines mêmes, visant à en renouveler les démarches.

Un exemple du premier cas est donné par une recherche-action, menée par deux enseignantes du cycle secondaire supérieur et centrée sur la figure de l'étranger ${ }^{24}$. Sur la base d'un échantillon de textes littéraires et à travers l'élaboration d'outils de décodage de la catégorie examinée, les enseignantes ont produits deux unités didactiques portant respectivement sur la figure de l'étranger dans la littérature de l'époque classique à nos jours et dans la réalité contemporaine. L'étude des cultures classiques comme cultures autres, rappelle Alessandra Durino Allegra, constitue le point de départ pour la réflexion sur le dynamisme des cultures, ainsi que sur leurs rencontres, conflits ou fusions ${ }^{25}$; réflexion que l'on voit bien se prolonger sur d'autres terrains disciplinaires, comme les langues étrangères ou l'histoire.

24 Nombre d'exemples de la deuxième démarche pourraient également être décrits, issus de l'enseignement de l'histoire, de la géographie, de la philosophie ou de la littérature.

\section{Culture(s) : les nouvelles frontières}

L'enseignement littéraire, notamment, constitue aujourd'hui un lieu de rencontre et d'opposition entre «deux cultures différentes »: la culture officielle, classique et essentiellement écrite, représentée par l'enseignant et la culture de masse, principalement médiatique, des jeunes. Dans une perspective interculturelle, l'enseignement littéraire se définit comme "une éducation littéraire respectueuse des droits de ses destinataires (et) qui se propose comme un processus de médiation et de dialogue entre deux types de culture et de goût $»^{26}$.

La frontière générationnelle, rappelait récemment Louis $\operatorname{Porcher}^{27}$, semble aujourd'hui se creuser : pour la première fois, dans notre civilisation, les grands-parents ne vivent plus avec les petits-enfants, l'urbanisation ayant mis fin à cette coexistence. Par ailleurs, on constate, dans les grandes villes, qu'il devient de plus en plus fréquent que les enfants 
rentrent à la maison plus tôt que les parents, qu'ils y assument des tâches domestiques et des responsabilités qui amènent à redéfinir autrement leur processus d'autonomisation. Pour la première fois, on parle, à propos des jeunes, de " générations pelliculisées ", c'està-dire rendues de plus en plus petites, de plus en plus fines: des pratiques générationnelles frontalisées se manifestent de plus en plus rapidement, réduisant l'écart qui sépare une génération de la suivante ; on se demande désormais s'il n'est pas question de frontière générationnelle entre des enfants de dix et des enfants de douze ans... Force est de constater, poursuivait Louis Porcher, que si l'école ne prend pas en compte cette évolution, elle commet une négligence qui risque, à terme, de se révéler suicidaire, puisque les processus en cours ont un impact sur l'attitude des élèves vis-à-vis de l'école, des savoirs que l'école leur transmet, des critères d'évaluation que les enseignants adoptent, critères qui n'ont pas forcément évolué au rythme du public auquel ils sont appliqués.

Ainsi, l'éducation à la différence, dans sa variante italienne, semble apte à prendre en compte également la frontière qui tendrait à séparer, au sein d'une même société, deux groupes d'âge qui affirment leur identité par des modalités socioculturelles, des « habitus » comme les appellerait Pierre Bourdieu, très différents, voire conflictuels.

Une autre discipline, dans le système éducatif italien, apporte sa contribution au projet commun d'une éducation à la diversité. Enseignants et élèves italiens ont tendance à considérer l'art italien comme l'expression inégalée de la production artistique mondiale ou, en tout cas, à privilégier spontanément les conventions culturelles et iconiques que l'art italien leur a rendues familières. Les normes esthétiques de sa propre civilisation assument naturellement le statut de références absolues et hégémoniques. Or, éduquer à l'art signifie comprendre que la diversité de ses manifestations ne se situe pas sur une échelle hiérarchiquement organisée ${ }^{28}$ : l'educazione interculturale, suivant l'approche bipolaire connaissance/compréhension citée précédemment, amène à acquérir «la connaissance des répertoires iconiques et des systèmes de conventions linguistiques et figuratives propres à chaque civilisation et, en même temps, la compréhension de la relation qui existe entre le système artistique et tous les autres systèmes qui, s'articulant avec l'art, contribuent à déterminer ses caractéristiques et son identité culturelle $»^{29}$.

On remarque que le cadre théorique sous-jacent à l'élaboration de ces approches est celui de l'anthropologie: la culture n'est-elle pas précisément considérée, par Claude LéviStrauss, "comme un ensemble de systèmes symboliques au premier rang desquels se placent le langage, les règles matrimoniales, les rapports économiques, l'art, la science, la religion. Tous ces systèmes visent à exprimer certains aspects de la réalité physique et de la réalité sociale et, plus encore, les relations que ces deux types de réalités entretiennent entre eux et que les systèmes symboliques eux-mêmes entretiennent avec les autres ${ }^{30} »$ ?

Une éducation à la différence et à la diversité humaines pourrait-elle d'ailleurs se passer de l'apport des anthropologues à la connaissance de nos manières de percevoir, identifier et caractériser l'Autre?

«Nous devons incontestablement à Lévi-Strauss, écrit Letizia Caronia, une représentation de la différence culturelle qui ne se résout ni à l'identification des étapes d'un développement intellectuel de l'humanité, ni à l'examen pointu de modèles culturels particuliers et incommensurables. (...) La valeur culturelle indéniable de la proposition de Lévi-Struass réside dans le fait d'avoir écarté toute présomption de supériorité d'une culture sur une autre et d'avoir témoigné, à travers son œuvre, de la dignité profondément humaine de tout produit culturel, en tant que manifestation de Yesprit humain ${ }^{31}$. " 
Mais, outre cette conscience - dont nous savons qu'elle est loin aujourd'hui d'être acquise - que faire du savoir des anthropologues, se demande Letizia Caronia, à l'heure où les anthropologues mêmes interrogent radicalement le statut de leur réflexion et nous transmettent une image inquiète, et inquiétante, de leur questionnement?

Ce que dans une perspective pédagogique nous pouvons raisonnablement demander à l'anthropologie, affirme-t-elle, c'est «l'exemple d'une construction de connaissance à partir de l'interaction. A ce propos, l'anthropologie fonde notre espoir d'accéder à une compréhension négociée de l'autre (spécifique et contextuelle) à travers des procédures dialogiques et un affinement continuel des pratiques de traduction ${ }^{32}$ ». Autrement dit, conclut-elle, ce qui est pertinent dans une réflexion pédagogique n'est pas le « quoi » du savoir des anthropologues mais le "comment»: c'est de leur métier, plus que de leur savoir, qu'un éducateur a besoin ${ }^{33}$. Pour comprendre la culture d'un enfant marocain, ce n'est pas le compte rendu ethnographique sur la culture du Maroc qu'un éducateur doit rechercher, mais les modalités, les procédures qui ont permis à l'anthropologue de produire un portrait culturel ${ }^{34}$.

\section{Les mots qui (dé)-classent}

33 Ainsi, le processus à travers lequel on perçoit et on catégorise l'Autre se situe au cœur des préoccupations que les ethnologues partagent avec les éducateurs, dans un souci, également commun, de contrôler et de dépasser la vision ethnocentrique, c'est-à-dire la tendance à hiérarchiser les cultures et leurs représentants en fonction de leur adéquation à nos propres critères, aussi relatifs qu'inconscients.

Mais l'école, se demande Vittoria Cesari, peut-elle valoriser ce que la société, de fait, dévalorise ${ }^{35}$ ? Pour les Latins, nomina sunt homina ${ }^{36}$ : relever les manières dont une société nomme les autres signifie non seulement identifier la représentation qu'elle s'en fait, mais également analyser les rapports de force que ces dénominations instituent tout en les révélant. Dans nos dictionnaires, nombre de termes sont fortement marqués par l'histoire des relations conflictuelles entre deux communautés ou relèvent de la réduction d'un groupe humain à un objet ou à une fonction (macaroni, par exemple, pour désigner les Italiens ou rosbif pour les Anglais). Les exemples de modalités de dénigrement sont nombreux, notamment dans la dénomination des groupes en situation de migration. Comme pour le mot «barbare » dans la Grèce antique, un « qualificatif d'altérité ${ }^{37}$ » peut renvoyer à l'« excentricité » de la langue parlée par une communauté étrangère : bougnat, par exemple, aphérèse de charbougna, nous informe Le Petit Robert, est une «imitation plaisante du parler des Auvergnats", marchands de charbons à Paris. En italien, l'expression vu'cumprà, qui désigne les immigrés non-européens (surtout Africains et en situation irrégulière), dérive de la phrase utilisée par ceux-ci, dans un italien approximatif, pour vendre leur marchandise (vuoi comprare?: veux-tu acheter?). En italien toujours, bien que le qualificatif de extracomunitari indique en apparence tous les étrangers n'appartenant pas à la Communauté européenne, il désigne, de fait, surtout ceux qui proviennent d'Afrique ou d'un des pays dits du tiers-monde. Dans cette catégorie, soulignent justement Patrick Johnson et Elisabetta Nigris ${ }^{38}$, ne sont pas inclus les ressortissants du Japon ou des États-Unis, alors que ces pays se situent bien au-delà des frontières européennes. Ainsi, les manières de nommer les ressortissants d'un pays étranger faisant référence à des catégories apparemment « objectives »- géographiques, 
en l'occurrence - ne sont pas moins tributaires de la représentation qu'une communauté se fait de l'étranger et des hiérarchies qu'elle y impose. Dans le terme d'extracomunitari s'additionnent donc les marques de la différence linguistique et culturelle, voire ethnique, mais aussi de la stigmatisation de pays exclus de l'aire de référence et des individus appartenant à une catégorie socio-économique défavorisée.

La valorisation d'un groupe étranger, dans une communauté donnée, est fonction du statut de ce groupe à l'intérieur de la communauté, mais elle dépend également de la manière dont la même communauté se situe, sur le plan géopolitique et culturel, par rapport à celles qui l'entourent. Plus nous avons emprunté à une société, plus nous avons partagé d'intérêts avec elle, écrit Lévi- Strauss, plus elle nous semble active. Une société qui semble active, "progressive ", fait l'objet d'une représentation positive, alors que, à l'inverse, on aura tendance à déprécier les sociétés qui nous semblent "stationnaires ${ }^{39}$ ".

Or, l'Italie, comme le rappelle la circulaire ministérielle citée plus haut, est aujourd'hui la destination de flux migratoires en provenance du Sud - essentiellement africain et nordafricain - et de l'Est - européen ou asiatique - alors que l'attirance s'intensifie, en Italie, pour les modèles culturels du Nord européen et de l'Ouest américain. Au-delà des liens commerciaux, politiques ou historiques «objectifs» - que l'émigration italienne a d'ailleurs contribué à tisser les pays d'Europe ou d'Amérique du Nord bénéficient aujourd'hui du statut valorisant que leur confère le fait de représenter, pour la communauté italienne, les sociétés "progressives" auxquelles on s'identifie et avec lesquelles on souhaite partager davantage d'intérêts.

L'évolution de l'enseignement des langues étrangères n'est-elle pas un indice supplémentaire de ces «affinités électives » de l'Italie avec les cultures notamment du Nord-Ouest atlantique?

Comme le rappelait Francesca Berté dans cette revue même, l'enseignement précoce des langues, après une phase d'expérimentation, a été introduit dès 1985 dans les programmes scolaires italiens ${ }^{40}$. Outre les objectifs cognitif et communicatif fixés, ces programmes incitent à une prise de conscience de l'intérêt de l'apprentissage des langues pour la compréhension des autres peuples et pour la valorisation du pluralisme linguistique et culturel. On ne sera pas surpris d'apprendre, cependant, que $82,07 \%$ des classes ont choisi l'anglais et qu' " un travail intense de persuasion sur le terrain » a dû accompagner l'introduction des autres langues (pour 13,5\% des classes le français, pour $2,95 \%$ l'allemand et pour $1,47 \%$ l'espagnol ${ }^{41}$ ).

\section{Savoir être... étranger}

Il est clair alors que si l'école ne veut pas rendre vains les efforts de son entreprise interculturelle, de sa vocation à l'educazione alla mondialità comme ouverture à la différence, à la pluralité des langues, des valeurs et des cultures, une question s'impose : l'adoption de cette finalité n'exige-t-elle pas une nouvelle conception de l'enseignement linguistique, qui mettrait tout d'abord en cause l'alignement du dispositif scolaire sur une demande sociale ayant un tel degré d'uniformité ?

40 L'Italie n'est évidemment pas le seul pays européen à se mesurer à cette situation. Cependant, compte tenu justement de cette aspiration quasi exclusive de la société italienne aux modèles d'une aire culturelle, il nous semble que si le système éducatif ne 
faisait que se conformer à cette logique, il satisferait davantage le circuit fermé d'une éducation mono culturelle que sa propre vocation au dépassement de l'ethnocentrisme.

Par ailleurs, bien que l'enseignant de langue ne puisse, seul, assumer cette responsabilité, n'est-ce pas dans la classe de langue étrangère que l'étranger, précisément, est introduit en tant que tel dans la dénomination même de la discipline ? Nous soulignerons, à la suite de Geneviève Zarate, que la classe de langue est « un des lieux où la culture du pays de l'élève et la culture étrangère enseignée entrent en relation" et que "les outils d'enseignement des langues vivantes présentent un intérêt sociologique particulier : ils mettent à plat la relation à l'étranger qu'une société donnée veut offrir en modèle à ceux qu'elle éduque $»^{42}$.

Adaptant cette définition de la classe de langue au contexte italien, nous ajouterons qu'elle est plus précisément le lieu où «les » cultures des élèves et «les » nouvelles cultures étrangères entrent en relation, bien que l'éducation à la diversité, quelle que soit la discipline dans laquelle elle s'inscrit, concerne autant les enfants natifs que les autres, la classe de langue étrangère est la seule, nous semble-t-il, où peuvent être valorisées et exploitées, au même titre, les compétences aussi bien des natifs diglossiques que des petits migrants bi- ou trilingues. Et ceci, d'autant plus s'il est vrai, comme l'écrit Danièle Moore, que «la valorisation du bilinguisme des enfants migrants par l'école ne nuit pas, au contraire, au développement de l'enseignement des langues étrangères, et contribue de surcroît à renforcer les valeurs positives accordées à la langue d'accueil ${ }^{43}$ ".

Et peut-être, revenant à l'exemple de Jorgu et Violeta une conciliation pourrait se réaliser avec une langue maternelle dont la valeur est si tributaire du regard de l'Autre. Dans le cadre de leurs activités scolaires, raconte Maria Teresa Romanello, Jorgu et Violeta rédigent chacun le portrait d'une personne aimée. On constate, chez eux comme chez tous les autres petits Albanais suivis par la même équipe d'enseignants, la même absence, celle de l'albanais, leur langue maternelle, qu'aucun d'entre eux n'a choisi. Ce qui constitue peut-être, selon Maria Teresa Romanello, un indice manifeste d'une volonté d'intégration sociale et linguistique. Les deux textes, rédigés donc en italien, sont représentatifs de deux niveaux différents de maîtrise de la langue écrite, tels que l'on peut retrouver également chez des élèves natifs. Les deux rédactions sont équivalentes quant à leur force descriptive, mais elles divergent sur le plan de la correction grammaticale, Violeta disposant d'outils syntaxiques, morphologiques, textuels plus efficaces et mieux contrôlés.

Á quelques mois de distance, la même revue fait état de la surprenante découverte que Violeta avait en réalité copié son texte. Ainsi, une autre compétence est à ajouter aux précédentes, puisque cette élève témoigne d'une étonnante capacité à extrapoler des phrases d'un texte pour en reconstruire un autre, sinon « original », du moins cohérent et parfaitement adapté aux attentes scolaires de correction et d'acceptabilité. «Reste suspendu - écrit Maria Teresa Romanello - le jugement sur l'acceptabtabilité "éthique" d'un tel procédé.» Mais c'est là que s'arrête, probablement, conclut-elle, la tâche du linguiste.

C'est là que commence et se justifie, nous semble-t-il, le défi de l'educazione interculturale. 


\section{NOTES}

1. Louis Porcher, «Lever de rideau ", Notions en Questions. Rencontres en didactique des langues, $\mathrm{n}^{\circ} 2$, janvier 1997, p. 19.

2. Marc Augé, Le sens des autres. Actualité de l'anthropologie, Paris, Fayard, 1994, p. 10.

3. «La domanda centrale per un educazione interculturale è : come pensare la differenza in un modo cherenda possibile la convivenza?», Letizia Caronia, «Pedagogia e differenze culturali : risorse e dilemmi del sapere degli antropologi », in: Elisabetta Nigris (sous la direction de), Educazione interculturale, Milano, Bruno Mondadori, 1996, p. 76.

4. Gerald et Silvia Arlettaz, « Les processus nationaux de stigmatisation de l'étranger. Constats et questions de recherche en histoire ", in: Cristina Allemann-Ghionda (sous la direction de), Multikultur und Bildung in Europa. Multiculture et éducation en Europe, Bern, Peter Lang, 1994, p. 171-179, p. 175-176.

5. Il s'agit de : Fulvio Poletti (sous la direction de), L'educazione interculturale, Firenze, La Nuova Italia, 1992.Alessandra Durino Allegra (sous la direction de), Verso una scuola interculturale, Firenze, La Nuova Italia,1993. Elisabetta Nigris (sous la direction de), Educazione interculturale, Milano, Bruno Mondadori, 1996.

6. Fulvio Poletti, op.cit, p. 119.

7. D'après Alessandra Durino Allegra, op.cit, p. 67.

8. Il s'agit plus précisément de la Circolare Ministeriale ${ }^{\circ} 122$ du 28 avril 1992 qui accompagne la Pronuncia du Consiglio Nazionale della Pubblica Istruzione du 23/4/1992, in : Alessandra Durino Allegra, op.cit, p. 185-195.

9. Ibid., p. 187.

10. Ibid., p. 188.

11. Alessandra Durino Allegra, op.cit, p. 68.

12. Voir dans le $n^{\circ} 1,1992$, de la revue Italiano \& Oltre p. 41-43 et le commentaire de Raffaele Simone, p. 34. Il s'agit de protéger les langues et cultures frioulanes et sardes, les langues et cultures des populations d'origine albanaise, catalane, germanique, grecque, slave, tzigane, ainsi que celles qui parlent le ladin, le français, le franco-provençal et l'occitan. À celles-ci il faut ajouter le français et l'allemand parlés dans deux régions qui bénéficient d'un statuto speciale comme le Val d'Aoste et le Trentino-Alto Adige. Effectivement, le projet du législateur est ambitieux, sinon irréaliste, voire tardif, comme l'affirme Raffaele Simone.

13. Ibid., p. 4.

14. Alessandra Durino Allegra, op.cit, p. 29.

15. Nous reprenons ici les données présentées dans Lorenzo Coveri, «Chi parla dialetto in Italia? », Italiano\& Oltre, $\mathrm{n}^{\circ} 5,1995, \mathrm{p} .198-202$.

16. Nous faisons référence aux articles de Maria Teresa Romanello, «L'italiano dei piccoli albanesi », paru dans Italiano \& Oltre, $\mathrm{n}^{\circ} 3,1995$, p. 134-137, et « Violeta farà carriera », Italiano \& Oltre, $\mathrm{n}^{\circ}$ 5, 1995, p. 274-275.

17. Fulvio Poletti, op.cit, p. 105.

18. Ibid.

19. Ibid., p. 143-144.

20. Ibid., p. 139.

21. Ibid., p. 143-144.

22. Ibid., p.XXI-XXII. 
23. Nous n'entrons pas ici dans le détail des diverses mesures et réformes préconisées pour réaliser le projet.

24. Cf. L Grossi, R. Rossi, « Per un educazione interculturale. Un'ipotesi didattica ", in : Notiziario Intercultura, n² 26, luglio-settembre 1991, cité par Alessandra Durino Allegra, op.cit., p. 152-153.

25. Alessandra Durino Allegra, op.cit, p. 152-153.

26. G. Armellini, «Insegnamento letterario ed educazione alla pace: appunti per una discussione ", in: AA.W., Lezioni di pace, CEDIP, Bologna, Thema, 1987, p. 219-237; cité par Alessandra Durino Allegra, op.cit., p. 153-155.

27. Nous résumons ici les propos de Louis Porcher, d'après les notes prises au cours de son intervention dans le cadre du séminaire «Frontières culturelles et diffusion des langues : quels enjeux éducatifs ?", dirigé par Geneviève Zarate, à l'École normale supérieure de Fontenay/ Saint-Cloud, 19/11/1996.

28. Alessandra Durino Allegra, op.cit, p. 160-161.

29. L. Lazotti Fontana, «Educare all'arte per favorire gli scambi culturali », in : La pedagogia degli scambi interculturali, Quaderno $n^{\circ} 10$ del CEDE, a cura di M.G.Calasso, Frascati, I Quaderni di Villa falconieri, 1986, p. 81 ; cité par Alessandra Durino Allegra, op.cit, p. 161.

30. Claude Levi-Strauss, «Introduction à l'œuvre de Marcel Mauss » (1950), in : Marcel Mauss, Sociologie et anthropologie, Paris, PUF, 6e éd. (Quadrige), p.XIX.

31. Letizia Caronia, « Pedagogia e differenze culturali... ", in : Elisabetta Nigris, op.cit., p. 42-43.

32. 32 Ibid., p. 68.

33. 33 Ibid., p. 69.

34. Ibid., p. 70.

35. Vittoria Cesari, «La dimensione interculturale nell'éducazione : riflessioni e riferimenti per l'azione pedagogica », in : Fulvio Poletti, op.cit, p. 95.

36. $C f$. Patrick Johnson et Elisabetta Nigris, «La questione dei termini », in: Elisabetta Nigris (a cura di), Educazione interculturale, Milano, Bruno Mondadori, 1996, p. 1-16.

37. Voir Marie Françoise Basiez, L'Étranger dans la Grèce antique, Paris, Les Belles Lettres, (Realia),1984.

38. Patrick Johnson et Elisabetta Nigris, «La questione dei termini », in : Elisabetta Nigris (a cura di), Educazione interculturale, Milano, Bruno Mondadori, 1996, p. 14.

39. Voir Claude Levi-Strauss, Race et histoire, Unesco, 1952, réed. Coll. Folio/Essais, 1987.

40. Francesca Berte, "Expérimentations italiennes ", in : Revue Internationale d'éducation-Sèvres. Les langues vivantes à l'école, $\mathrm{n}^{\circ}$ 9, mars 1996, p. 113-118.

41. Ibid., p. 117-118

42. Geneviève Zarate, Représentations de l'étranger et didactique des langues, Paris, Didier, Coll. «Credif-Essais », 1993, p. 11.

43. Danièle Moore, «L'école et les représentations du bilinguisme et de l'apprentissage des langues chez les enfants ", in : Cristina Allemann-Ghionda (sous la direction de), Multikultur und Bildung in Europa.Multiculture et éducation en Europe, op.cit, p. 125-138, p. 137. 


\section{RÉSUMÉS}

L'immigration est un thème nouveau pour l'Italie. Mais l'expérience cruciale de la diversité italienne et d'une unité patiemment conquise met en valeur l'éducation interculturelle et la formation à la compréhension et au respect de la différence.

INDEX

Index géographique : Italie

Mots-clés : éducation interculturelle, minorité linguistique, diversité linguistique, immigration

\section{AUTEUR}

\section{GLORIA PAGANINI}

Attachée temporaire d'enseignement et de recherche, section sciences de l'éducation, École normale supérieure de Fontenay-Saint-Cloud 